\title{
PERFORMANCE OF THE P-BAND SUBSYSTEM AND THE X-BAND INTERFEROMETER OF THE F-SAR AIRBORNE SAR INSTRUMENT
}

\author{
A. Reigber, M. Jäger, M. Pinheiro, R. Scheiber, P. Prats, J. Fischer, R. Horn, and A. Nottensteiner \\ Microwaves and Radar Institute, German Aerospace Center (DLR), Germany
}

\begin{abstract}
The F-SAR airborne SAR instrument represents the successor of the E-SAR system of the German Aerospace Center (DLR), which had previously been extensively used over the last three decades. Its development was triggered by the current demand for simultaneous acquisitions at different wavelengths and polarisations as well as by the demand for very high resolution in the order of decimetres. F-SAR is a modular development utilising state of the art hardware. As for E-SAR, DLRs Dornier DO228-212 aircraft is the first choice as platform for the new system.

With the recently completed X-band single-pass interferometer and the P-band subsystem, F-SAR is now ready for fully replacing the E-SAR. This paper presents the two new subsystems and analyses their performance based on fully polarimetric imagery acquired during recent system test and calibration campaigns.
\end{abstract}

Index Terms - Synthetic aperture radar, airborne sensors

\section{F-SAR INSTRUMENT DESIGN AND STATUS}

The new airborne SAR instrument F-SAR has been operated by DLR since 2009 as the successor to the former E-SAR system. As with the E-SAR sensor, which was operational between 1983 and 2010, F-SAR is intended as a technology test-bed to establish expertise in SAR system design, signal processing and image analysis. The F-SAR instrument has been entirely designed and constructed at DLR, using commercial-off-the-shelf components where possible. Design-critical parts however are developed and built inhouse. Like the former E-SAR system, F-SAR is installed and operated on-board DLR's Dornier DO228-212 aircraft as the platform of choice.

F-SAR is designed to operate fully polarimetrically at X-, C-, S-, L- and P-bands and will provide single-pass polarimetric interferometric capabilities in X- and S-band. Repeatpass PolInSAR is a standard measurement mode for the other bands. Range resolution is determined by the available system bandwidth. While components limit system bandwidth to $100 \mathrm{MHz}$ at P-band, a step-frequency approach is adopted to achieve up to $760 \mathrm{MHz}$ effective signal bandwidth at $\mathrm{X}$ band to satisfy the requirement for very high resolution. An overview of the general design parameters can be found in Tab. 1.

F-SAR's main design feature is the fully polarimetric operation in five frequency bands (X-, C-, S-, L- and P-band) with the ability to measure different frequency bands and/or polarisations simultaneously in four recording channels. Furthermore, the system design features two single-pass polarimetric across-track interferometers (XTI) in X- and S-band. Currently, all subsystems are completed and undergoing an optimisation of their performance. While the X-, C-, S- and L-band system have already been operated in flight for some time, the P-band system was just recently completed.

For regular Earth observation purposes the radar covers an off-nadir angle range of 25 to 60 degrees at altitudes of up to $6000 \mathrm{~m}$ above sea level, which is the maximum operating altitude of the DO228 aircraft. In special applications, other off-nadir angle ranges, such as 60 to 85 degrees for long stand-off imaging or 0 to 25 degrees for sounding or steep incidence applications are feasible.

\section{EXPERIMENTAL RESULTS}

\subsection{The P-band subsystem}

F-SAR's recently completed P-band subsystem features a switchable centre frequency of $350 \mathrm{MHz}$ (P1-band) or $435 \mathrm{MHz}$ (P2-band). In both sub-bands, certain limitations concerning the bandwidth arise from constraints imposed by the problematic frequency allocation at P-band. The lower

Table 1. F-SAR technical characteristics.

\begin{tabular}{|l|ccccc|}
\hline & $\mathrm{X}$ & $\mathrm{C}$ & $\mathrm{S}$ & $\mathrm{L}$ & $\mathrm{P}$ \\
\hline \hline RF [GHz] & 9.6 & 5.3 & 3.25 & 1.325 & $0.35 / 0.45$ \\
Bw [MHz] & 760 & 400 & 300 & 150 & $100 / 50$ \\
max PRF [kHz] & 5 & 5 & 5 & 10 & 10 \\
PT [kW] & 2.5 & 2.2 & 2.2 & 0.9 & 0.9 \\
Rg res. [m] & 0.2 & 0.4 & 0.5 & 1.0 & 1.5 \\
Az res. [m] & 0.2 & 0.3 & 0.35 & 0.4 & 1.5 \\
Rg cov. [km] & \multicolumn{5}{|c}{ 3-5 (at max. bandwidth) } \\
Sampling & 4 bit real, 1000MHz \\
RX channels & 4, 2 per band \\
Data rate & \multicolumn{5}{|c}{ MByte/s (per channel) } \\
\hline
\end{tabular}


P1-band is entirely blocked by NATO frequencies and can usually only be used for civil applications outside of NATO territory. It is, however, relatively unaffected by RFI. The upper P2-band is better accessible, but strongly affected by various external radiation sources. In case of F-SAR, a maximum bandwidth of $100 \mathrm{MHz}$ is possible at $\mathrm{P} 1$-band, while only $50 \mathrm{MHz}$ are realised for P2-band: bandpass filters in the receive chain are needed to reduce RFI influences. The P-band antennas of F-SAR are planar array antennas and are mounted under the nose of the aircraft. Currently, only an antenna for the P1-band is available, which is identical to the former antenna used with the E-SAR. A new antenna for P2-band is under development. For both bands, the transmit power lies around 900W maximum.

The maiden flight of F-SAR's P-band system, in cooperation with the University of Zurich, took place the 26th March 2012 over the test-site of Vordemwald in Switzerland. During this campaign, the bandwidth had to be limited to $20 \mathrm{MHz}$ due to possible interference with the glideslope instrument landing system and the Swiss public safety communication systems, both operating within the P1-band. The data acquisitions included the recording of intermediate listen-only pulses, fully polarimetric acquisitions and several repeat-pass baselines.

Fig. 1 shows a fully polarimetric P-band image acquired during this campaign. The data were processed to an azimuth resolution of 1 metre, resulting in a comparatively good image quality despite the low range resolution. The $1.5 \mathrm{~m}$ corner reflectors deployed in the scene turned out to be too small at this resolution level for proper analysis of image resolution and radiometric accuracy. Further tests are required to precisely calibrate the system.

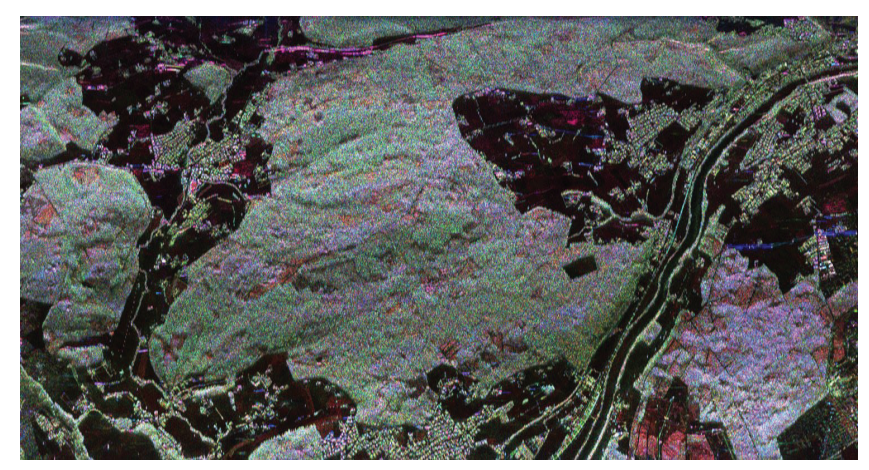

Fig. 1. Fully polarimetric P-band image acquired by F-SAR over the test-site of Vordemwald/Switzerland. (blue: VV polarisation, red: HH polarisation, green: HV polarisation).

However, these initial system tests already allow the estimation of the noise equivalent sigma zero (NESZ) level of the P-band subsystem. Fig. 2 top shows the coherence $\gamma_{x x}$ between the two cross-polar channels HV and VH. For reciprocal media both should be identical, apart from uncorrelated noise components in the receiver chain. Under this assump- tion, an estimate of NESZ can be derived from the coherence and the local intensity $I$ by $N E S Z=I \cdot\left(1-\gamma_{x x}\right)$. At the bottom of Fig. 2, an estimated range profile of F-SAR's NESZ performance is plotted, revealing excellent values around -50 to $-55 \mathrm{~dB}$. It has to be noted, however, that different radar parameter settings, as well as a different flight altitudes, may result in significantly different values, both higher or lower.

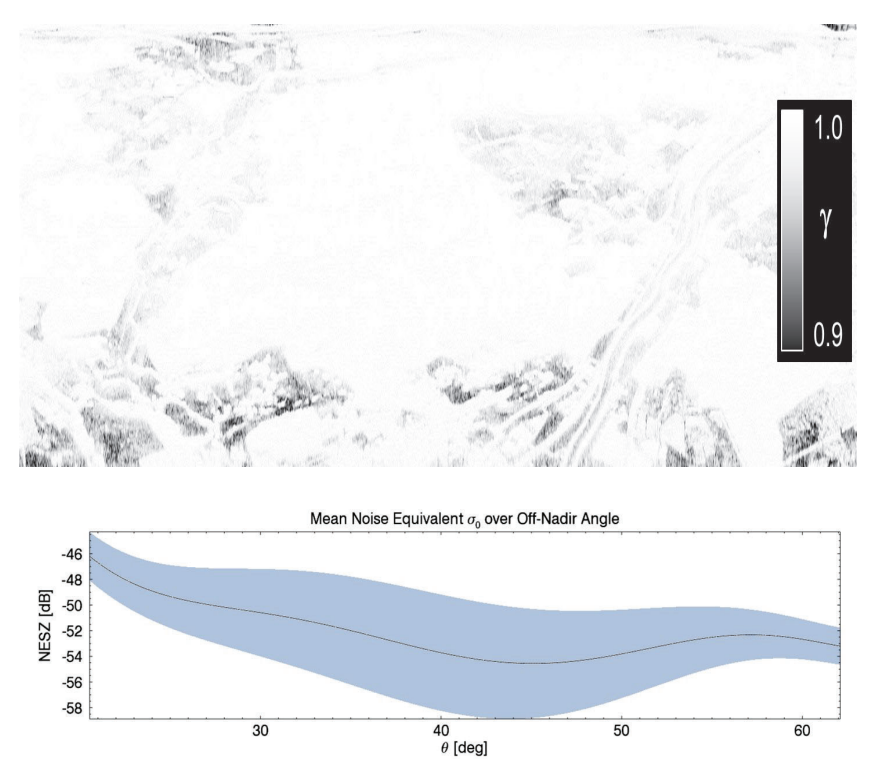

Fig. 2. Analysis of F-SAR's system noise level at P-band. Top: Coherence between HV and VH channels. Bottom: Estimated noise equivalent sigma zero values.

The first P-band test also featured polarimetric repeat-pass interferometry. The top of Fig. 3 shows the topographic phase after flat-earth compensation for a baseline of about $10 \mathrm{~m}$. Despite the hilly terrain, only few topographic fringes occur due to the long wavelength and comparatively small baseline. Notably are the high-quality phase measurements obtained even over forested regions. At the bottom of Fig. 3, the polarimetric coherence of the scene is shown, depicting the varying decorrelation levels in the different polarimetric channels. Over strongly vegetated areas, coherence is very high, while open ground shows a reduced coherence. This is currently thought to be due to the lower signal level in these areas combined with the limited opposite swath suppression due to the mount position of the P-band antenna below the fuselage.

\section{2. $X$-band interferometry}

FSAR's X-band subsystem features 6 antenna ports, that can be used to simultaneously mount and operate 3 dual-polar antennas. With the 2 available receiver chains and pulse interleaved switching between the antenna ports, various transmit / receive configurations can be realised. In standard imaging mode, spatial resolutions of up to $25 \mathrm{~cm}$ in fully polarimetric mode are possible. This configuration has been operational 


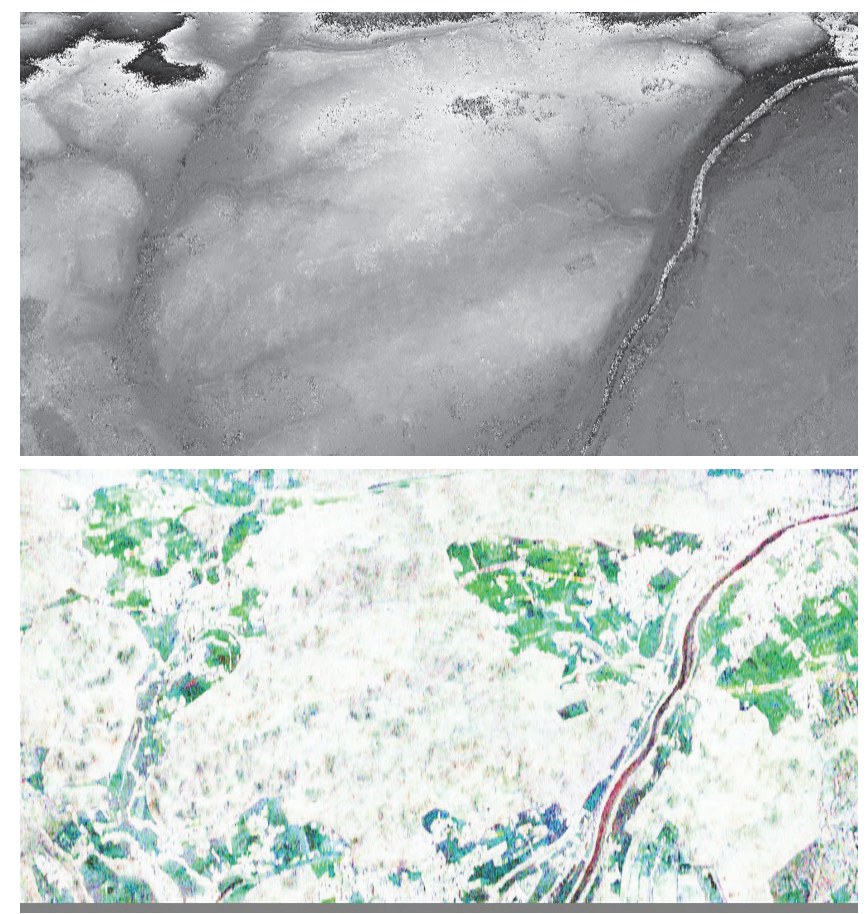

Fig. 3. P-band repeat-pass interferometry. Top: Repeat-pass phase at $10 \mathrm{~m}$ baseline (VV-polarisation). Bottom: PolInSAR coherence (blue: VV decorrelation, red: HH decorrelation, green: $\mathrm{HV}$ decorrelation)

since 2008 using an intermediate experimental antenna configuration.

For F-SAR's final configuration, a special antenna carrier was designed to fix several planar array antennas to the aircraft. In fully-fledged multi-frequency configuration, it holds seven right-looking dual polarised antennas: three at X-band, one at C-band, two at S-band and one at L-band. The antenna carrier has the important advantage of making it easy to change the antenna configuration and to mount other antennas without necessitating additional individual airworthiness certification procedures. This nominal antenna configuration provides, among others, an across track (XTI) and an along track (ATI) interferometer at X-band. The mechanical baselines are approx. $1.6 \mathrm{~m}$ (XTI) and approx. $85 \mathrm{~cm}$ (ATI). With this configuration, single-pass PolInSAR data acquisitions become possible. Fig. 4 shows the antenna carrier with the three $\mathrm{X}$-band antenna positions.

The X-band single-pass polarimetric interferometer had its maiden flight shortly after the P-band subsystem on April 6th 2012 over the test-site Kaufbeuren / Germany and some days later over the Jade Bight on the German North Sea coast. Fig. 5-8 present some first results of these campaigns. In Fig. 5, the PolSAR X-band image acquired by antenna X1 (see Fig. 4) at a resolution of $0.5 \mathrm{~m}$ is shown. Fig. 6 presents the single-pass interferometric phase in VV polarisation after flat-earth removal. Finally, Fig. 7 depicts the PolInSAR co-

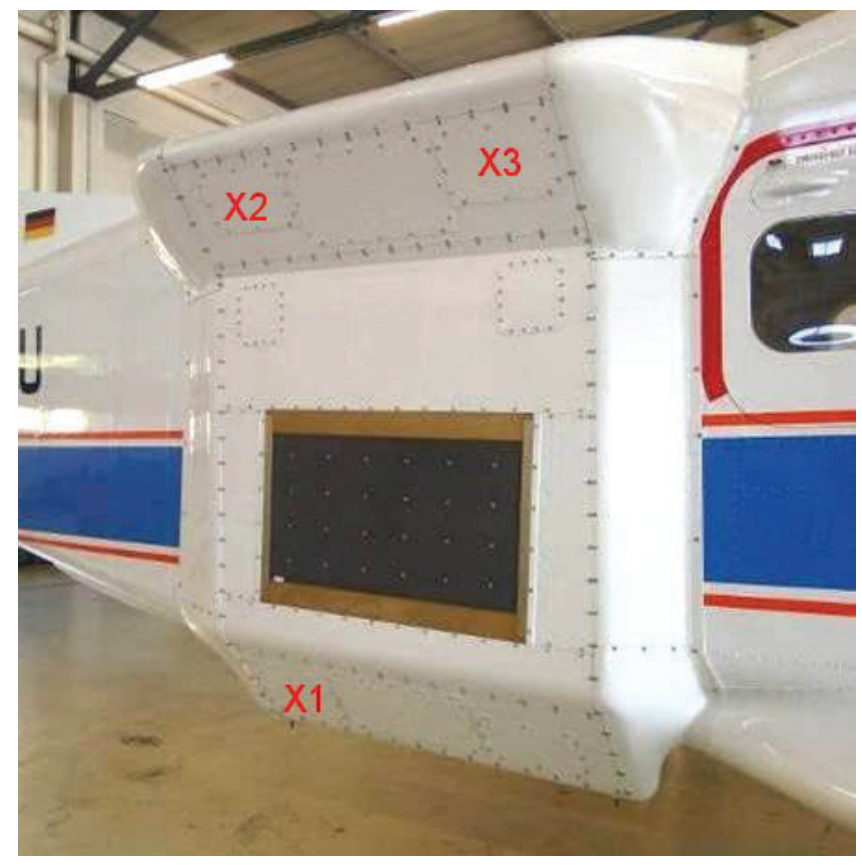

Fig. 4. F-SAR antenna carrier. The 3 positions of the X-band antennas for XTI and ATI operation are marked as X1, X2 and $\mathrm{X} 3$.

herence. The large buildings left of the scene centre caused some saturation effects particularly in the HH channel. Also, the low cross-polar backscattering of the runway in the middle of the scene leads to SNR decorrelation. Both effects are caused by the inaccurate amplifier settings, which were made manually during this first system test. An automatic gain control will be used in future campaigns. Up to now there have been no attempts to derive and evaluate precise digital elevation models from these data. This task requires a precise calibration of the baseline and antenna behaviour and is the scope of ongoing work.

Fig. 8 shows a zoom of phase and coherence values obtained over a flat costal area crossed horizontally by many shallow drainage channels and two vertical dikes of several metres height. The fine drainage channels are well visible at the resolution of $0.5 \mathrm{~m}$, the coherence is as high as expected in the single-pass case. Again, it was not tried to derive and evaluate precise digital elevation models from these data. However, these first system test of the X-band interferometer are very promising concerning F-SAR's future DEM quality.

\section{CONCLUSIONS}

The paper summarises the system status, calibration and current performance of the F-SAR instrument. The results demonstrate that the F-SAR is close to be ready for operational purposes in all its frequency bands from $\mathrm{X}$ to $\mathrm{P}$. Generally, the system shows an excellent performance in all 


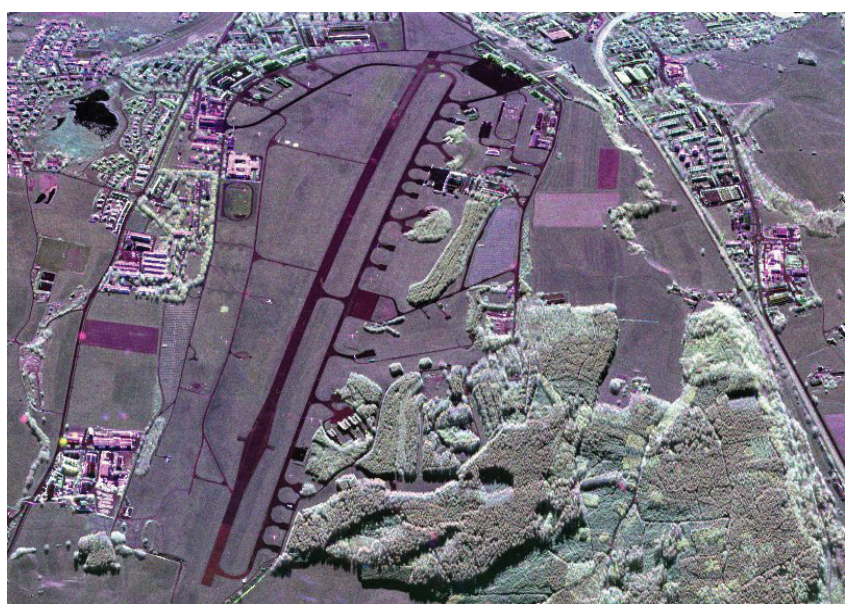

Fig. 5. X-band single-pass polarimetric interferometric data acquisition by F-SAR, polarimetric image result (blue: VV polarisation, red: HH polarisation, green: HV polarisation).

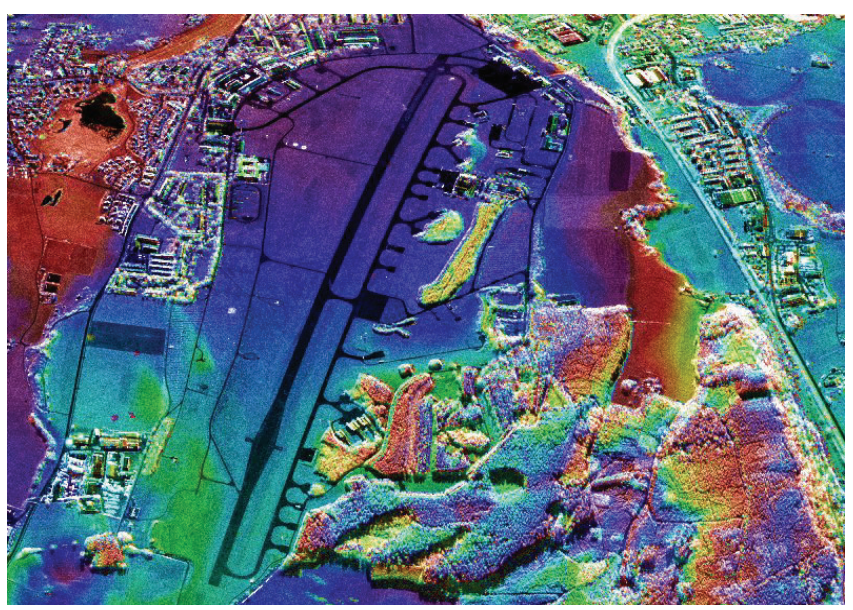

Fig. 6. X-band single-pass interferometry, overlay of flattened interferometric phase with VV amplitude.

bands although fine calibration of various components is still ongoing.

The availability of fully polarimetric single-pass interferometric SAR data at X-band, and soon also at S-band, opens new possibilities for future research in the field of polarimetric interferometry. It is expected that these data will deliver important insights for the development of new information products as well as for the design of future satellite missions, such as TanDEM-L.

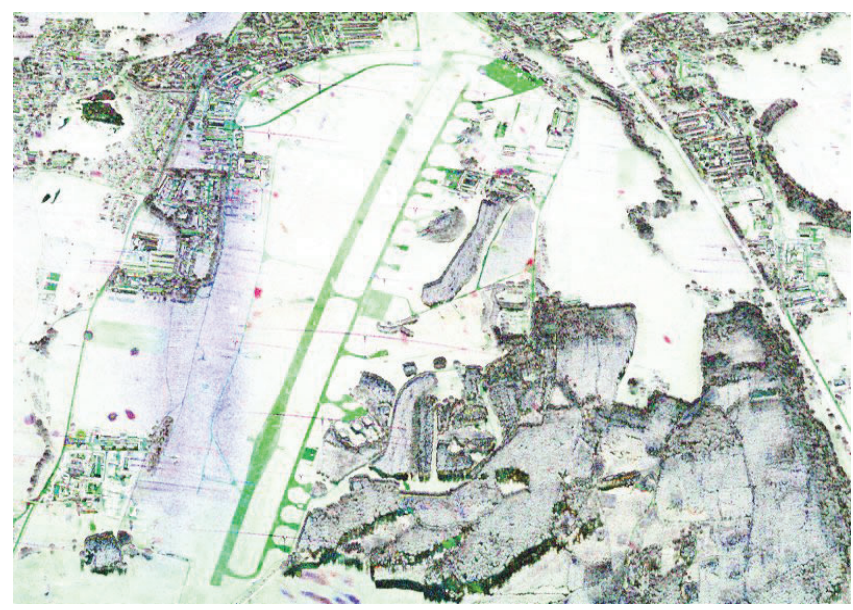

Fig. 7. X-band single-pass interferometry, PolInSAR coherence (blue: VV decorrelation, red: HH decorrelation, green: HV decorrelation).
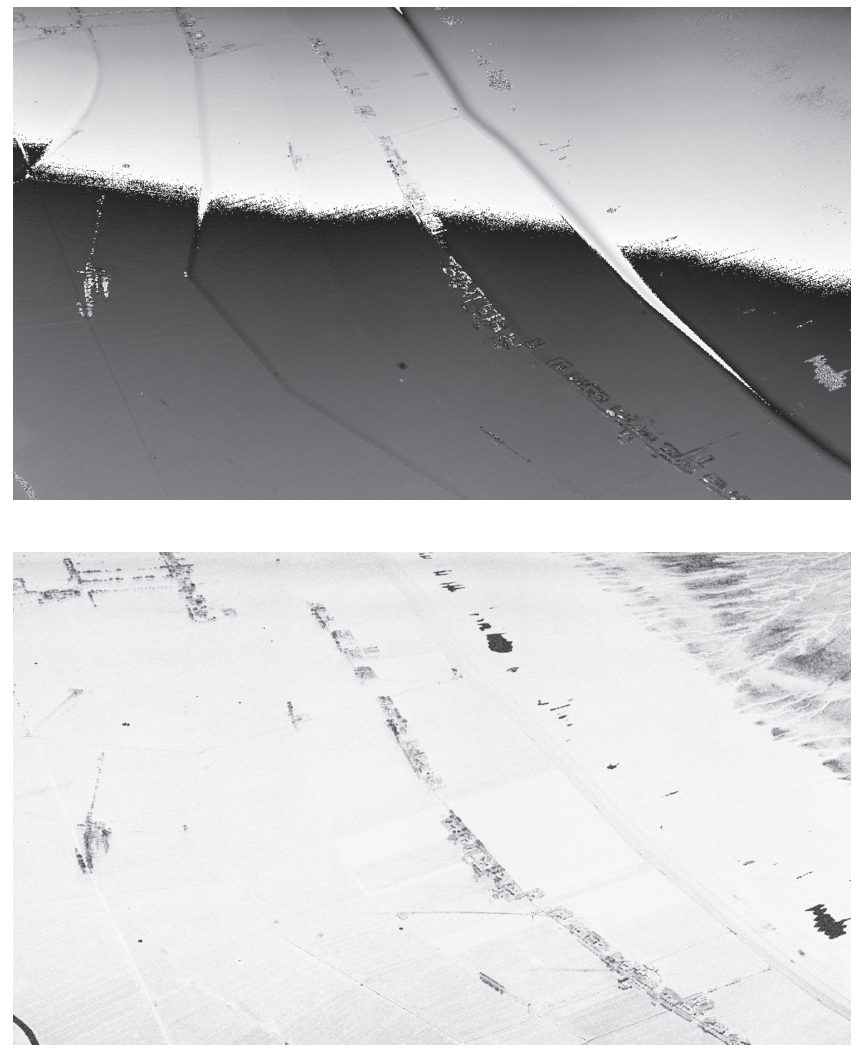

Fig. 8. Test-site Jade Bight / Germany: Zoom into the Xband single-pass interferometric phase (top) and coherence (bottom), HH polarisation. 